Article available at nttp://Www.parasite-journal.org or nttp://dx.dol.org/10.1051/parasite/2002093233

\title{
Une nouvelle pUCE du GenRe Allopsylla Beaucournu et Fain, 1982 (SipHonaptera : IschNopsyllidae) en République Centrafricaine, sur Un Chiroptère Molossidae méconNu
}

\author{
BARRIÈRE P.*, BEAUCOURNU J.C.**, MÉNIER K.*** \& COLYN M.*
}

Summary: A NEW FLEA SPECIES OF THE GENUS ALLOPSYLLA Beaucournu et Fain, 1982 (Siphonaptera: Ischnopsyllidae) From Central African Republic, on a pOORLy KNOWN molossid bat A pair of Allopsylla lobayensis Beaucournu \& Barrière, new species were collected the first of November 1999 in Ngotto forest, in the south-western part of the Lobaye basin, Central African Republic (CAR). The host specimen, a little known molossid bat (Myopterus whitleyi), constitutes the first record of this species in CAR. The new flea species appears taxonomically close to A. hetera Beaucournu \& Fain, 1982, one of the two other Allopsylla species, previously know from other faunal regions (A. alloides in Nigeria and A. hetera in Democratic Republic of three known Allopsylla species raises questions regarding the hostparasite specificity of this group.

KEY WORDS : Allopsylla lobayensis sp. n., Siphonaptera, Ischnopsyllidae, Myopterus whitleyi, Ngotto forest, Central African Republic. the Congo). The parasitism of three distinctly different hosts by the

\begin{abstract}
Résumé :
Un couple d'Allopsylla lobayensis Beaucournu et Barrière sp. n. a été récolté le ler novembre 1999 en forêt de Ngotto, dans la partie sud-ovest du bassin de la Lobaye, République Centrafricaine (RCA). Le spécimen hôte, un chiroptère molossidé méconnu (Myopterus whitleyil, constitue la première collecte de l'espèce en RCA. La nouvelle puce s'apparente à $\mathrm{A}$. hetera Beaucournu et Fain, 1982, l'une des deux autres espèces du genre Allopsylla connues jusqu'alors de régions fauniques distinctes (A. alloides au Nigéria et A. hetera en République Démocratique du Congo). L'identification de trois hôtes parmi les trois espèces d'Allopsylla interpelle sur la spécificité hôte-parasite au sein de ce groupe.
\end{abstract}

MOTS CLÉS : Allopsylla lobayensis sp. n., Siphonaptera, Ischnopsyllidae, Myopterus whitleyi, forêt de Ngotto, République Centrafricaine.

sodique, 12 microchiroptères dont un molossidé porteur de deux puces appartenant à une espèce nouvelle.

\section{HÔTE (CHIROPTERA: MOLOSSIDAE)}

\section{MYOPTERUS WHITLEYI (SCHARFF, 1900)}

dans le bassin fluvial de la Lobaye, à l'extrême nord du domaine forestier guinéo-congolais, la zone d'étude de Batouri $\left(03^{\circ} 54^{\prime} \mathrm{N}-17^{\circ} 02^{\prime} \mathrm{E}\right)$ se situe dans la partie occidentale de la forêt classée de Ngotto. La couverture végétale y est de type forêt primaire semi-caducifoliée non climacique. En plus de l'échantillonnage mensuel des rongeurs et musaraignes, la première année de suivi a permis à l'un de nous $(\mathrm{PB})$ de capturer, de façon épi-

\footnotetext{
* Laboratoire Éthologie-Évolution-Écologie, UMR 6552 CNRS, Station Biologique (SBP), Université de Rennes 1, 35380 Paimpont, France. Tél. : $33(0) 299618162$ - Fax : 33 (0)2 99618188. E-mails: Patrick.Barriere@univ-rennes1.fr; Marc.Colyn@univ-rennes1.fr ** Laboratoire de Parasitologie et Zoologie appliquée, Faculté de Médecine, 2, avenue du Pr Léon Bernard, 35043 Rennes Cedex, France. Tél. : 33 (0)2 23234643 - Fax : 33 (0)2 23234629 .

E-mail : jbeaucou@univ-rennes1.fr

*** Laboratoire de Parasitologie Pharmaceutique, Faculté de Pharmacie, 2, avenue du Pr Léon Bernard, 35043 Rennes Cedex. Tél. : 33 (0)299650301 - Fax : 33 (0)2 99301534 .

E-mail: KMenier@ugpvb.fr

Correspondance: Patrick Barrière.
}

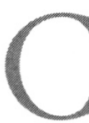
ette chauve-souris (réf. SBP: BA0312) a été capturée vivante le $1^{\text {er }}$ novembre 1999 , au début de la saison sèche, sur une ligne de pitfall installée au sol pour la collecte des musaraignes. Le seau de capture situé à $70 \mathrm{~m}$ du bord de la rivière Batouri est inclus dans la ligne P1 (session de piégeage "Octobre 1999, XIII (48)" in Barrière \& Nicolas (2000)). La collecte au sol de ce chiroptère est d'autant plus surprenante que le spécimen ne présentait aucune blessure apparente et que l'espèce semblerait, selon Cosson (comm. pers.), inféodée à la canopée forestière. De plus, ce spécimen constitue la première collecte de l'espèce en RCA (Carte 1). Les deux puces prélevées sur ce chiroptère font l'objet de cette description.

\section{ALLOPSYLLA LOBAYENSIS \\ BEAUCOURNU ET BARRIÈRE SP. N.}

Matériel examiné : holotype mâle, allotype femelle, sur Myopterus whitleyi (Scharff, 1900) (hôte $\mathrm{n}^{\circ} \mathrm{BA} 0312$, col- 


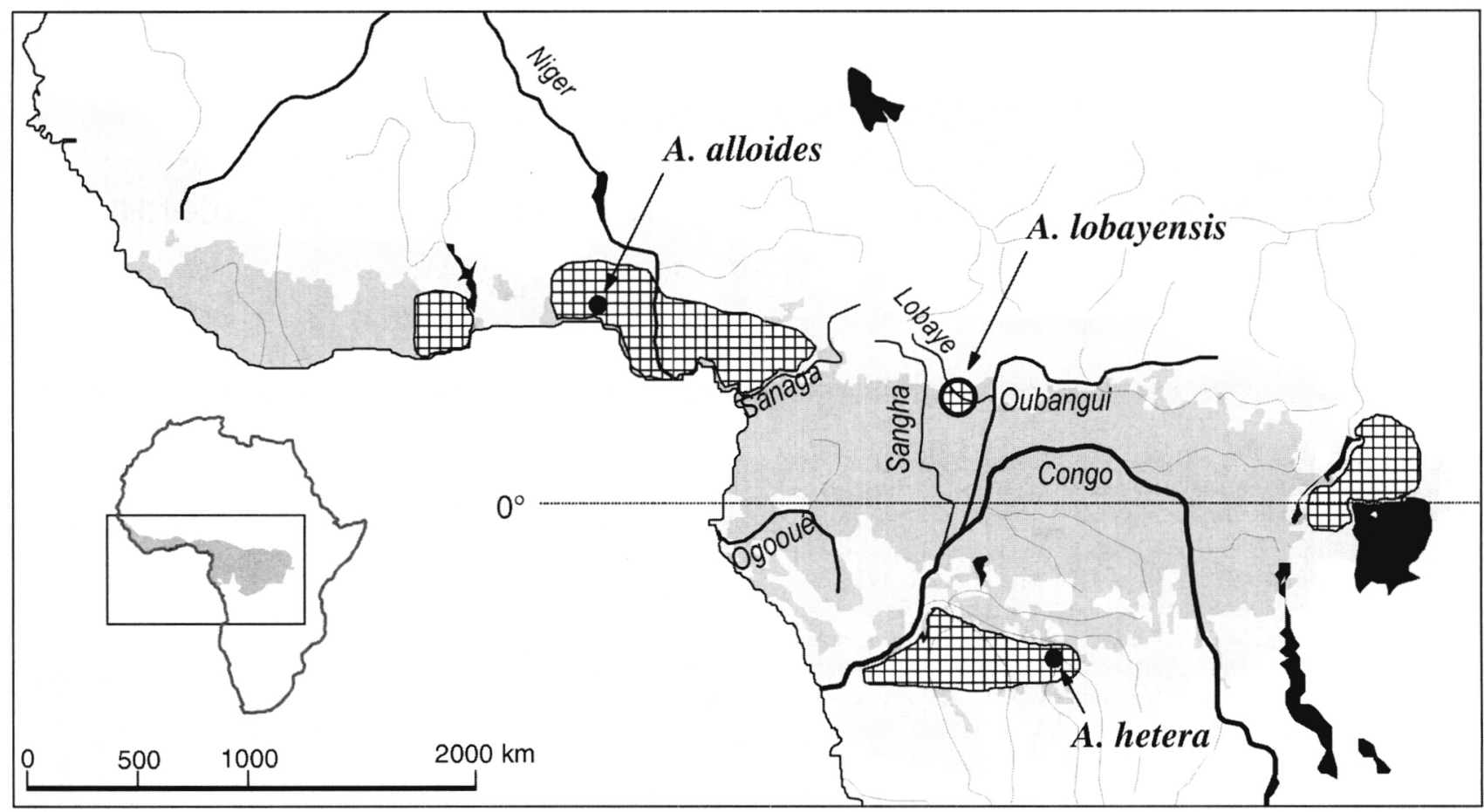

Bloc forestier guinéo-congolais

Zone de savane incluant des isolats forestiers

\# Distribution probable de Myopterus whitleyi

Site d'étude : Batouri, Forêt de Ngotto, république Centrafricaine

Carte 1. - Distribution estimée de Myopterus whitleyi et sites de collecte des trois espèces d'Allopsylla au sein du domaine forestier guinéocongolais.

lection M.C., Station Biologique de Paimpont puis déposé au Laboratoire de Zoologie : Mammifères \& Oiseaux du Muséum National d'Histoire Naturelle, Paris sous la référence CG 2001-1834), Batouri (035 $54^{\prime}$ $\mathrm{N}-17^{\circ} 02^{\prime} \mathrm{E}$ ), forêt de Ngotto, République Centrafricaine, $1^{\text {er }}$ novembre 1999.

Dépôt des types : actuellement dans la collection J.C.B., Rennes et ultérieurement déposés au Laboratoire d'Entomologie du Muséum National d'Histoire Naturelle, Paris.

Derivatio nominis: le nom de cette espèce est une latinisation du nom de la rivière (Lobaye) qui arrose la forêt de Ngotto (zone d'intervention du programme ECOFAC) où furent collectés l'hôte et les deux puces.

\section{Description}

Espèce proche d'Allopsylla hetera Beaucournu \& Fain, 1982. Nous ne donnerons que les caractères les plus typiques du genre et ceux qui la différencient d'A. hetera et $A$. alloides (Smit, 1977), les deux seules espèces jusqu'alors connues dans le genre.

Capsule céphalique : très proche de celle d'Allopsylla hetera dont elle se distingue (sur le nombre limité de spécimens connus : un couple de chaque espèce) par le léger chevauchement de la base des deux épines de la cténidie génale. Le rétrécissement de l'apex de la première épine est présent (figures 1-2). Beaucournu \& Fain (1982) l'avaient signalé à propos d'A. hetera mais le dessin était peu explicite et c'est pourquoi nous en donnons ici une nouvelle représentation (figure 3).

Thorax : prothorax portant une cténidie de 24 épines acuminées chez le mâle, 26 chez la femelle (il y en a respectivement 20 chez $A$. alloides dont seul le mâle est connu et 28 chez A. hetera (mâle et femelle types)).

Mésothorax : une pseudosetae sur le mesonotum chez le mâle (au tiers supérieur) et deux chez la femelle; mesosternum portant une soie ventrale chez le mâle, deux chez la femelle : il y en a deux chez $A$. alloides et une chez le mâle et la femelle d'A. hetera, mais si la présence de soie à cet emplacement est importante pour la diagnose générique, il ne faut pas attacher une signification trop précise à leur nombre, la chétotaxie étant toujours un peu variable. Metanotum avec quatre spinules marginales; metasternum sans soie ventrale chez le mâle, avec une (ou deux ?) soie(s) chez la femelle.

Abdomen (segments non génitaux) : chetotaxie classique; respectivement 2-1-1-1 spinules sur la marge des 

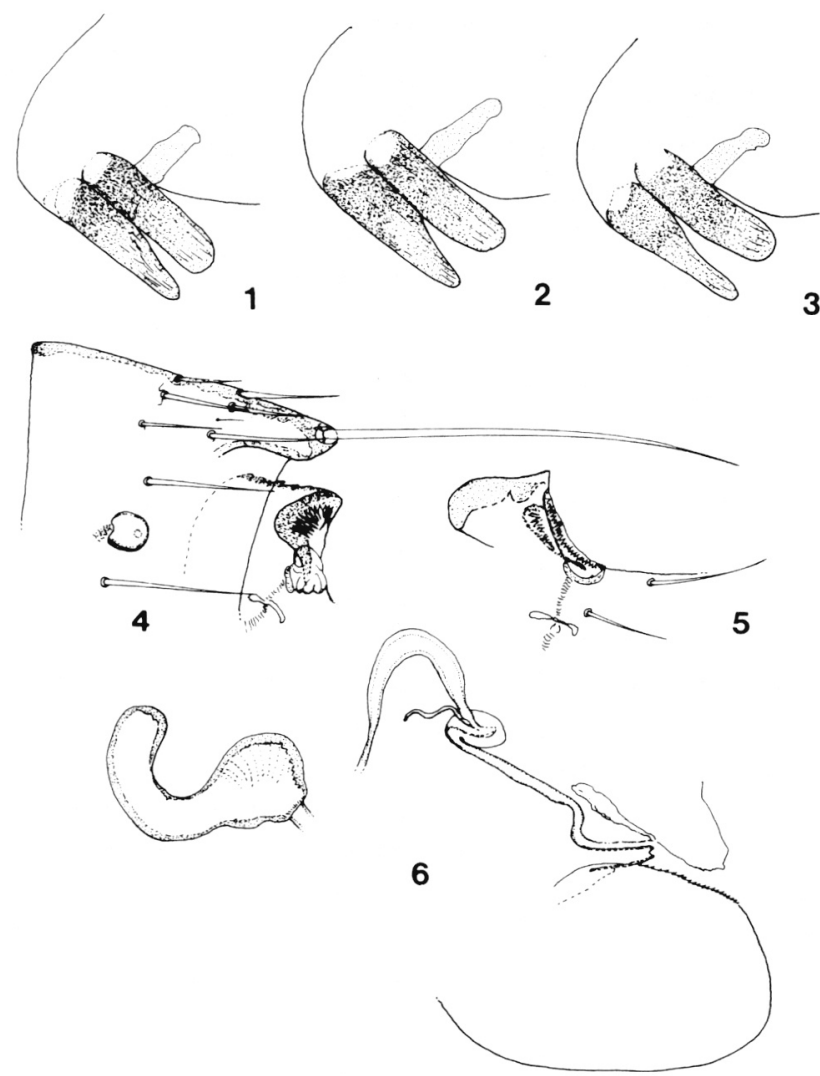

Figures 1 à 6. - Allopsylla spp.

1 à 3 : cténidie génale, respectivement holotype et allotype d'A. lobayensis sp. n., et holotype d'A betera; $4:$ A. lobayensis, partie des tergites VII et VIII, soie antesensiliale et stigmate de l'allotype: 5: A. lobayensis, partie du tergite VIII et stigmate de l'holotype ; 6 : A. lobayensis, sternite VIII (partim), spermathèque et ducti (partim) de l'allotype.

tergites I à IV dans les deux sexes. Soie antesensiliale unique et longue dans les deux sexes (nous figurons ici celle de la femelle (figure 4) qui était cassée chez l'allotype d' $A$. hetera). Stigmates du metepimeron et des tergites grands et circulaires comme chez $A$. hetera (ils sont nettement plus petits chez A. alloides). Le stigmate du tergite VIII est très aberrant, semblant former un double spiracle (femelle: figure 4 ; mâle : figure 5). Nous n'avons observé ceci ni chez les autres Allopsylla, ni chez aucun autre siphonaptère. Si mâle et femelle ne présentaient, aux différences sexuelles près, les mêmes structures symétriques, nous aurions eu tendance à les classer comme stigmates tératologiques ce qui est peu fréquent mais existe chez les puces (Beaucournu et al., 1999) comme chez les autres insectes. Tergite II femelle portant en plus de la soie ventrale classique, deux soies latérales courtes mais bien visibles sur la face latérale (il n'y en a pas chez le mâle, comme chez ceux des autres Allopsylla, mais nous rappelons que la femelle d'A. alloides demeure inconnue).

Segments génitaux du mâle et phallosome. Tergite IX : basimère (figure 7) immédiatement séparé de celui

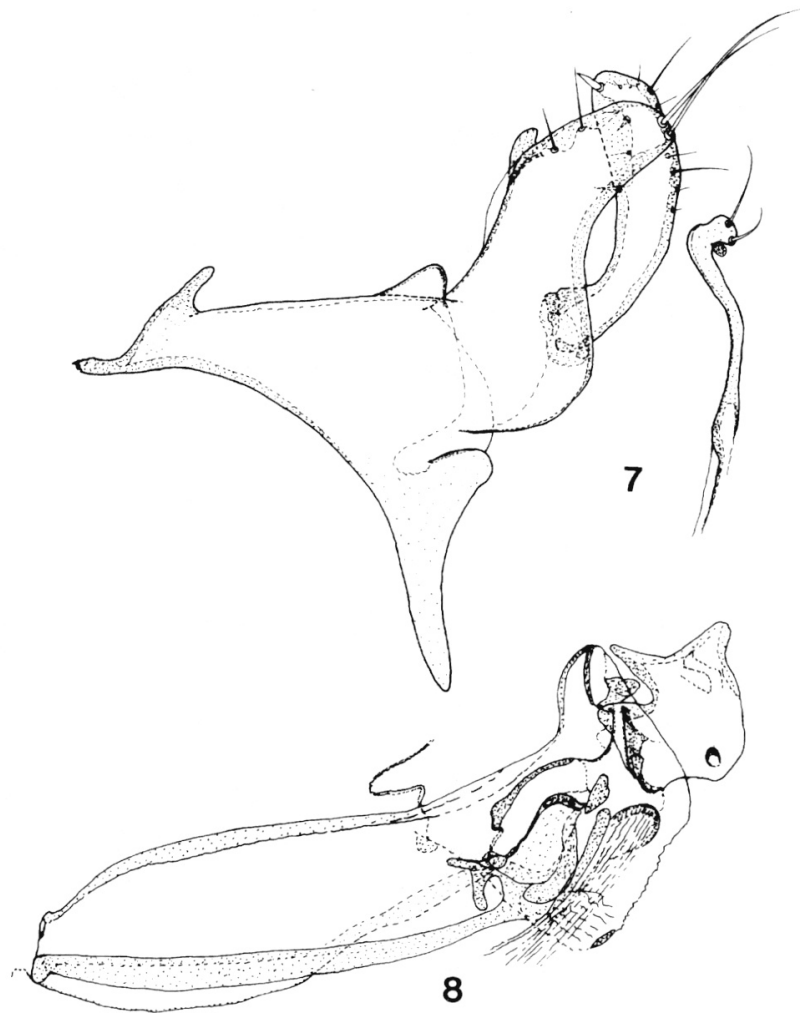

Figures 7 et 8 . - Allopsylla lobayensis sp. n., holotype. 7 : segment IX; 8 : phallosome.

d'A. alloides par le fait que cette partie du tergite est courbée vers l'arrière et non subrectiligne, et d'A. hetera par sa largeur, plus faible. L'expansion submarginale interne de la marge dorsale du basimère existe comme chez $A$. hetera mais est plus étroite et située plus antérieurement. Télomère plus grêle, plus arqué et plus saillant au-dessus de l'apex du basimère que chez $A$. hetera; il est nettement différent chez $A$. alloides. Sternite IX (figure 7) réduit à sa partie distale comme il est de règle dans ce genre et recourbé en crosse, mais ici la face dorsale de cette "crosse" est concave et non convexe. Par ailleurs, la chétotaxie des trois espèces est analogue. Le phallosome (figure 8) est très apparenté, ce qui est normal, chez les trois Allopsylla. Le caractère le plus original chez $A$. lobayensis est l'aedeagus (figure 8), comme il est classique dans ce genre et les genres apparentés (Lagaropsylla et Araeopsylla) : il s'en distingue par la forme du "bec" rétrograde, ici moins effilé et la protubérance apicale qui est médiane et à distance du bord postérieur.

Segments génitaux de la femelle, ducti et spermathèque : le tergite VII est identique à celui d'A. hetera. Le sternite VII est malheureusement indiscernable dans la zone de sa marge postérieure : les rares segments semblant visibles évoquent ceux de l'espèce précédente. Le sternite VIII (figure 6) est original par la spinulation de son bord dorsal. Notons également (figure 6) 
l'absence du ductus communis, le ductus obturatus se séparant du $d$. spermathecae dès la perula. Le $d$. obturatus paraît beaucoup plus court que chez $A$. betera mais il ne s'agit, peut être, que d'un problème optique, l'apex étant perpendiculaire à l'axe de vision. Spermathèque (figure 6) non élargie à l'apex de la billa et la base de celle-ci ne montre pas d'étranglement comme chez $A$. hetera.

Dimensions (insectes montés) : holotype 1,9 mm, allotype $2,2 \mathrm{~mm}$.

\section{DISCUSSION}

11 llopsylla alloides a été capturée (holotype mâle) au Nigeria (Agege, $06^{\circ} 38^{\prime} \mathrm{N}-03^{\circ} 19^{\prime} \mathrm{E}$ ); A hetera (types) en République Démocratique du Congo, dans la région du Kasaï ( $\left.05^{\circ} 54^{\prime} \mathrm{S}-22^{\circ} 25^{\prime} \mathrm{E}\right)$ et $A$. lobayensis (types) enfin, en République Centrafricaine, dans le bassin de la Lobaye ( $\left.03^{\circ} 54^{\prime} \mathrm{N}-17^{\circ} 02^{\prime} \mathrm{E}\right)$. Ceci donne une répartition connue, de part et d'autre de l'équateur et à proximité de régions fauniques distinctes (Colyn \& Deleporte, sous presse), relativement réduite et allopatrique pour le genre Allopsylla. Le spécimen unique d'A. alloides est une collecte sur Molossidae : Tadarida (Mops) congicus Allen, 1917 (Bergmans rec. et det.), l'allotype d'A. hetera (l'holotype mâle est une collecte sur Galagoides demidoff (Primates: Galagonidae)) et les deux exemplaires d'A. lobayensis proviennent de la même espèce de molossidé, Myopterus whitleyi.

Selon Koopman (1993), M. whitleyi est présent au Ghana, Nigeria, Cameroun, République Démocratique du Congo et en Ouganda. Il était jusque-là inconnu dans la partie centrale et supérieure du bloc forestier congolais (Van Cakenberghe et al., 1999) et en RCA (Bergmans, 1978; Schlitter et al., 1982; Hill, 1983; Lunde et al., 2001). Le spécimen unique de cette chauve-souris récolté en forêt de Ngotto, constitue donc la première preuve de la présence de l'espèce en RCA et permet d'élargir sa distribution dans la partie septentrionale du bloc central (Carte 1). L'apparente rareté de ce molossidé (Van Cakenberghe et al., 1999) et le nombre réduit de spécimens collectés (Adam et al., 1993) sont probablement étroitement liés aux difficultés de capture et à son habitat situé dans la canopée (Cosson, comm. pers.). En raison de ces difficultés, nous ne connaissons probablement pas encore les limites exactes de sa distribution, mais il apparaît que cette espèce, bien que forestière (Rosevear, 1965; Hayman \& Hill, 1971), ait une distribution non contiguë et périphérique au bloc forestier congolais. Cette espèce semble d'ailleurs plus étroitement liée à la zone de mosaïque forêt-savane qu'à la forêt elle-même. Alors que ce microchiroptère est présent sur les trois sites de collecte d'Allopsylla, l'identification de trois hôtes
(Tadarida (Mops) congicus pour A. alloides, Galagoides demidoff pour A. hetera et Myopterus whitleyi pour $A$. hetera et $A$. lobayensis) interpelle sur la spécificité hôte-parasite au sein de ce groupe.

Malgré les similarités observées entre les deux espèces de siphonaptères d'Afrique centrale ( $A$. hetera et $A$. lobayensis), en terme d'espèce hôte et de morpho-anatomie, la complexité de la relation hôte-parasite au sein du genre Allopsylla confirme l'intérêt de ce groupe comme marqueur de l'histoire des faunes en Afrique tropicale.

\section{REMERCIEMENTS}

T 'étude sur le terrain a été financée par le programme européen (EU-DGVIII) Ecofac "Conserdvation et Utilisation Rationnelle des Ecosystèmes Forestiers en Afrique centrale, http://www.ecofac.org/". P. Barrière a reçu une bourse du Ministère de l'Éducation Nationale de l'Enseignement Supérieur et de la Recherche (France). Nous sommes particulièrement reconnaissant envers A. Pénelon (ECOFAC) pour son support logistique sur le terrain, les assistants de recherche (G. Yangoundjara et R.K. Maro) et les autorités du Ministère des Eaux, Forêts, Chasses et Pêches et du Tourisme à Bangui (RCA). Nous adressons également nos remerciements à $\mathrm{V}$. Van Cakenberghe (Belgique) pour l'identification spécifique du spécimen hôte et les données écologiques concernant l'espèce. Nous remercions enfin tout particulièrement le Dr E. De Coninck, section d'Entomologie, Musée Royal de l'Afrique Centrale à Tervuren, d'avoir bien voulu nous confier les types d'Allopsylla hetera, mis en dépôt dans son Institut.

\section{RÉFÉRENCES}

Adam F., Aellen V. \& Tranier M. Nouvelles données sur le genre Myopterus. Le statut de Myopterus daubentonii Desmarest, 1820 (Chiroptera : Molossidae). Revue Suisse de Zoologie, 1993, 100 (2), 317-326.

Barrière P. \& Nicolas V. Écologie et structuration des peuplements de micromammifères : Musaraignes et Rongeurs. Rapport d'expertise sur la Biodiversité animale en forêt de Ngotto (République Centrafricaine). ECOFAC-CEE, AGRECOCTFT, 2000, 55 p. (http://www.ecofac.org).

Beaucournu J.C. \& Fain A. Allopsylla hetera gen. n., sp. n. d'Afrique centrale (Siphonaptera, Ischnopsyllidae). Revue de Zoologie Africaine, 1982, 96, 559-569.

Beaucournu J.C., Gómez M.S. \& Ménier K. Apports de la tératologie à l'étude des Siphonaptères : discussion à propos de trois cas de stigmates surnumeraires. Boletin de la Asociacion española de Entomologia, 1999, 23, 249-256.

BERgmans W. Rediscovery of Epomophorus pousarguesi Trouessart, 1904 in the Central African Empire (Mammalia, Megachiroptera). Journal of Natural History, 1978, 12, 681-687. 
Colyn M. \& Deleporte P. Biogeographic analysis of Central African forest guenons, in: The guenons: Diversity and Adaptation of African monkeys. Glenn M. \& Cords M. (eds), Plenum Press, 6, 59-76, sous presse.

Hayman R.W. \& Hill J.E. Order Chiroptera, in: The Mammals of Africa. An identification Manual. Part 2. Meester J. \& Setzer H.W. (eds), Smithsonian Institute Press, Washington, 1971.

Hill J.E. Further records of bats from the Central African Republic (Mammalia: Chiroptera). Annals of the Carnegie Museum, 1983, 52, 55-58.

Koopman K.F. Order Chiroptera, in: Mammal species of the world, a taxonomic and geographic reference, Second edition. Wilson D.W. \& Reeder D.A. (eds), Washington D.C, 1993, 137-241.

Lunde D.P., Conway T.M. \& Beresford P. Notes on a collection of bats (Chiroptera) from Dzanga-Sangha, Central African Republic. Mammalia, 2001, 65, 535-540.

Rosevear D.R. The Bats of west Africa, Trustees of the British Museum (Natural History) London: i-vii, 1965, 418 p.

Schlitter D.A., Robbins L.W. \& Buchanan S.A. Bats of the Central African Republic (Mammalia: Chiroptera). Annals of the Carnegie Museum, 1982, 51, 133-155.

Smit F.G.A.M. A new bat-flea from Nigeria (Siphonaptera: Ischnopsyllidae). Beaufortia, 1977, 26, 39-42.

Van Cakenberghe V., De Vree F. \& Leirs H. On a collection of bats (Chiroptera) from Kikwit, Democratic Republic of the Congo. Mammalia, 1999, 63, 291-322.

Reçu le 6 mai 2002 Accepté le 6 juin 2002 